Clinical investigation

\title{
Potassium infusion increases the likelihood of conversion of recent-onset atrial fibrillation - a single-blinded, randomized clinical trial
}

Kiarash Tazmini, MD 1,2,3, Mai S. Aa. Fraz, MD', Ståle H. Nymo, MD, PhD¹, Mathis K. Stokke, MD,

PhDa3,4, William E. Louch, PhD2,3, Erik Øie, MD, $\mathrm{PhD}^{1,3}$

${ }^{1}$ Department of Internal Medicine, Diakonhjemmet Hospital, Oslo, Norway

2Institute of Experimental Medical Research, Oslo University Hospital, Ullevål and University of Oslo, Oslo, Norway

3K.G. Jebsen Center for Cardiac Research and Center for Heart Failure Research, University of Oslo, Oslo, Norway

4Clinic of Internal Medicine, Lovisenberg Diakonale Hospital, Oslo, Norway

Correspondence $^{1}$ : Kiarash Tazmini, MD, Department of Internal Medicine,

Diakonhjemmet Hospital, PO BOX 23 Vinderen, 0319 Oslo, Norway.

Email:kiakol3@gmail.com

${ }^{1}$ Current workplace: Department of Endocrinology, Morbid Obesity and Preventive Medicine, Faculty of Medicine, Oslo University Hospital, Postbox 4950 Nydalen, 0424 Oslo. Email: kiakol3@gmail.com 


\begin{abstract}
Background: The optimal antiarrhythmic management of recent-onset atrial fibrillation or atrial flutter (ROAF) is controversial and there is a considerable variability in clinical treatment strategies. It is not known if potassium infusion has the potential to convert ROAF to sinus rhythm (SR). Therefore, we aimed to investigate if patients with ROAF and plasmapotassium levels $\leq 4.0 \mathrm{mmol} / \mathrm{L}$ have increased probability to convert to SR if the plasmapotassium level is increased towards the upper reference range (4.1-5.0 mmol/L).

Methods: In a placebo-controlled, single-blinded trial, patients with ROAF and plasmapotassium $\leq 4.0 \mathrm{mmol} / \mathrm{L}$ presenting between April 2013 and November 2017 were randomized to receive potassium chloride $(\mathrm{KCl})$ infusion $(n=60)$ or placebo $(n=53)$. Patients in the $\mathrm{KCl}$ group received infusions at one of three different rates: $9.4 \mathrm{mmol} / \mathrm{h}(\mathrm{n}=11), 12$ $\mathrm{mmol} / \mathrm{h}(\mathrm{n}=19)$, or $15 \mathrm{mmol} / \mathrm{h}(\mathrm{n}=30)$.
\end{abstract}

Results: There was no statistical difference in the number of conversions to SR between the $\mathrm{KCl}$ group and placebo [logrank test, $P=0.29$; hazard ratio (HR) 1.20 (CI 0.72-1.98)].

However, KCl-infused patients who achieved an above-median hourly increase in plasmapotassium (>0.047 $\mathrm{mmol} / \mathrm{h}$ ) exhibited a significantly higher conversion rate compared with placebo [logrank $P=0.002$; HR 2.40 (CI 1.36-4.21)] and KCl patients with below-median change in plasma-potassium [logrank $P<0.001$; HR 4.41 (CI 2.07-9.40)]. Due to pain at the infusion site, the infusion was prematurely terminated in 10 patients (17\%).

Conclusions: Although increasing plasma-potassium levels did not significantly augment conversion of ROAF to SR in patients with potassium levels in the lower-normal range, our results indicate that this treatment may be effective when a rapid increase in potassium concentration is tolerated and achieved. 
Clinical Trial Registration -URL:https://clinicaltrials.gov. Unique identifier:

NCTo1818583

Key words: atrial fibrillation, potassium, antiarrhythmic drugs, treatment.

\section{Introduction}

Atrial fibrillation (AF) is the most common arrhythmia, with an estimated prevalence of approximately $3 \%$ in adults aged 20 years or older. Atrial flutter has lower prevalence, with an incidence $<1 / 10$ that of $\mathrm{AF}^{1}$. Recent-onset atrial fibrillation or atrial flutter (ROAF), defined as an abrupt onset of symptoms within 48 hours from admission, are highly prevalent in emergency departments ${ }^{2}$. Current European and American guidelines describe different treatment options and strategies for management of ROAF ${ }^{1}$. This is reflected by a wide variability of treatment approaches employed in clinical practice ${ }^{2}$. Indeed, common strategies range from a "wait and see" approach to conversion to sinus rhythm (SR) by use of anti-arrhythmic medication or direct-current cardioversion ${ }^{3}$.

About $50 \%$ of patients with ROAF with duration less than 48 hours convert spontaneously to SR 4,5. Several antiarrhythmic drugs have been shown to increase the likelihood of conversion to SR to about $70 \%$ within 12 hours ${ }^{6}$. However, most current antiarrhythmic drugs are not atrial specific, and they all have significant risk of side-effects, including atrial and ventricular proarrhythmia, heart failure exacerbation, lung-, hepatic-, and thyroid toxicity, and death 7. Furthermore, antiarrhythmic drugs have several contraindications including bradycardia, sinoatrial node dysfunction, AV conduction disturbances, prolonged QT interval, heart failure, coronary artery disease, and structural heart disease 7. By comparison, direct-current cardioversion has been shown to have a success rate of more than $90 \%$ in ROAF patients ${ }^{8}$. However, this approach requires deep sedation and a six hours postprandial period to ensure gastric emptying, associated with additional costs, resources, and risk.

Clinical studies have shown that lower serum concentrations of potassium $(<3.5 \mathrm{mmol} / \mathrm{L})$ are associated with a higher risk of $\mathrm{AF} 9$. This association is independent of age, sex, serum 
magnesium, antihypertensives, myocardial infarction, heart failure, and other potential confounders ${ }^{9}$. It has been suggested that hypokalemia may trigger AF through adverse effects on the sinoatrial node and increased pulmonary vein arrhythmogenesis ${ }^{10}$. Hypokalemia causes resting membrane hyperpolarization, $\mathrm{Na}^{+}-\mathrm{K}^{+}$ATPase inhibition and suppression of $\mathrm{K}^{+}$channel conductances resulting in action potential duration prolongation, reduced repolarization reserve, early afterdepolarizations, delayed afterdepolarizations, and automaticity. These changes are believed to increase the risk of developing AF ${ }^{11}$. Thus, increasing potassium intake with subsequently increased or normalized plasma-potassium levels might be beneficial in AF patients, mirroring previous work showing that this approach reduces both blood pressure and the risk of lethal ventricular arrhythmias in patients with ischemic heart disease, heart failure, and left ventricular hypertrophy ${ }^{12}$. There are several case reports of patients with AF converting to SR during hyperkalemia ${ }^{13-18}$. However, to our knowledge there are no studies investigating whether potassium infusion has the potential to convert AF to sinus rhythm. We presently hypothesized that patients with ROAF or atrial flutter with plasma-potassium levels $\leq 4.0 \mathrm{mmol} / \mathrm{L}$ have increased probability to convert to SR if the plasma-potassium level is increased towards the upper part of the reference range; between 4.1 and $5.0 \mathrm{mmol} / \mathrm{L}$. Thus, our aim was to investigate whether potassium, a cheap treatment with few contraindications, could be an alternative to more expensive drugs with potentially serious side-effects.

\section{Materials \& methods}

\section{Study design}

A single center, placebo-controlled, parallel-group study was conducted with equal randomization (1:1), blinded for patients but not for the healthcare providers, at Diakonhjemmet Hospital, Oslo, Norway, from April 2013 to November 2017. Diakonhjemmet Hospital is a local urban hospital in Oslo, Norway, serving approximately 135000 inhabitants. The patients are referred to the emergency department from general 
1 practitioners, municipal emergency services, nursing homes, community health services, or

2 directly by ambulance ordered from a dispatch center.

\section{Participants}

Eligible participants were patients $\geq 18$ years of age visiting the emergency department with $\mathrm{AF}$ or atrial flutter with abrupt onset of symptoms within 48 hours from admission and plasma-potassium $\leq 4.0 \mathrm{mmol} / \mathrm{L}$. Patients with or without a documented history of previous AF/atrial flutter were included. A 12-lead ECG was recorded (Mortara ELI 280, Mortara Instruments, Inc., Milwaukee, WI), at rest and interpreted by the treating physician. Exclusion criteria were kidney failure with estimated glomerular filtration rate (eGFR) <30 $\mathrm{mL} / \mathrm{min}$, current usage of antiarrhythmic medication (flecainide, amiodarone, dronedarone, sotalol, dofetilide or propafenone), infection, metabolic acidosis with $\mathrm{pH}<7.2$, pregnancy, or lactation. Patients participating in other clinical trials or who had previously been included in our trial were also excluded. Duration of symptoms, age, gender, body mass index (BMI), comorbidities and medications were registered shortly after patient inclusion, based on a review of medical records. After conversion to SR and before discharge, transthoracic echocardiography was performed, registering the following parameters: left ventricular (LV) ejection fraction, left atrial area, LV end-diastolic diameter (LVEDD), LV mass, mitral regurgitation, systolic pulmonary artery pressure (SPAP), and the LV diastolic parameters E/A-ratio (ratio between mitral valve early and late diastolic inflow) and E/e' (ratio between early mitral inflow velocity and mitral annular early diastolic velocity).

The study was approved by the Regional Committee for Medical and Health Research ethics, The Norwegian Medicines Agency, and the hospital's research committee, and was registered at Clinicaltirals.gov (NCTo1818583). All study patients provided their informed written consent. KT was funded by The South-Eastern Norway Regional Health Authority (Helse SørØst RHF); grant number: 2013011. Helse Sør-Øst RHF had no role in study design, data collection and analysis, decision to publish, or preparation of the manuscript. The authors are 
solely responsible for the design and conduct of this study, all study analyses, the drafting and editing of the paper and its final contents.

\section{Randomization, study treatment, and monitoring}

Patients were assigned to either the potassium chloride ( $\mathrm{KCl})$ group or placebo group following blocked randomization by shuffling opaque concealed envelopes. The $\mathrm{KCl}$ group was treated with $60 \mathrm{mmol} \mathrm{KCl}$ added to $1000 \mathrm{~mL}$ of $50 \mathrm{mg} / \mathrm{mL}$ dextrose in an infusion bag, which was then inverted ten times to ensure that the solution was well-mixed. $\mathrm{KCl}$ was administered through a peripheral venous catheter in a large vein, preferably the antecubital vein, at a rate of $166 \mathrm{~mL} / \mathrm{h}(9.4 \mathrm{mmol} \mathrm{KCl} / \mathrm{h}), 212 \mathrm{~mL} / \mathrm{h}(12 \mathrm{mmol} \mathrm{KCl} / \mathrm{h})$, or $265 \mathrm{~mL} / \mathrm{h}(15$ $\mathrm{mmol} \mathrm{KCl/h).} \mathrm{An} \mathrm{interim} \mathrm{analysis} \mathrm{was} \mathrm{performed} \mathrm{to} \mathrm{determine} \mathrm{whether} \mathrm{serum/plasma-}$ potassium increased as hypothesized. Since there was a smaller increase than expected, only the highest infusion rate was continued. Patients in the $\mathrm{KCl}$ group exhibiting serum magnesium levels $\leq 0.80 \mathrm{mmol} / \mathrm{L}$ also received magnesium sulfate infusion $(0.5 \mathrm{mmol} / \mathrm{kg}$ added to $1000 \mathrm{~mL}$ of $9 \mathrm{mg} / \mathrm{mL}$ sodium chloride) over 24 hours at a rate of approximately 42 $\mathrm{mL} / \mathrm{h}$.

$\mathrm{KCl}$ infusion was stopped if the patients converted to SR, the plasma-potassium level reached $\geq 5.5 \mathrm{mmol} / \mathrm{L}$, severe ventricular arrhythmia or symptomatic bradycardia occurred, eGFR declined to $<30 \mathrm{~mL} / \mathrm{min}$, or more than weak pain was experienced at the infusion site. The placebo group received only $50 \mathrm{mg} / \mathrm{mL}$ dextrose at the same infusion rate as in the $\mathrm{KCl}$ treatment group.

One of the trial investigators (KT) performed a day to day monitoring of potential adverse clinical events. Patients who did not convert to SR during infusion of $\mathrm{KCl}$ or placebo were treated by direct-current cardioversion the day after the start of infusion. The minimum infusion time for patients who did not convert to SR during infusion was 12 hours and the maximum infusion time was 24 hours. Patients with tachycardia (heart rate $>100$ beats $/ \mathrm{min}$ ) could receive rate-reducing treatment with a beta-blocker, a calcium-antagonist, digoxin or a combination of agents (beta-blocker + digoxin or calcium antagonist + digoxin) after the 
treating physician's assessment since these drugs are considered to have little converting properties and not regarded as antiarrhythmic drugs ${ }^{1}$.

Serum levels of potassium, magnesium, and creatinine (venipuncture) and plasma-potassium (arterial blood gas) were measured at inclusion. Serum-potassium, magnesium, and creatinine were further analyzed every 8 hours after the start of infusion and when the infusion was stopped, whereas plasma-potassium were measured 4 hours after start of infusion and thereafter every 8 hours, i.e. in between the serum analyses, and when the infusion was halted. Thus, serum- or plasma-potassium was monitored every 4 hours.

\section{Continuous ECG-monitoring by telemetry was performed on all patients.}

At the follow-up consultation performed roughly 3 months after discharge, a 12-lead ECG was recorded at rest, and all patients with SR were equipped with a hand-held "thumb ECG" recorder (Zenicor-EKG ${ }^{\circledR}$; Zenicor Medical Systems AB, Stockholm, Sweden) for 3 days. Patients were instructed to perform 30 second rhythm recordings once in the morning, once in the evening, and whenever they experienced palpitations or arrhythmia symptoms. The recorded signal corresponded to lead I in a standard 12-lead ECG. AF was defined as irregular ventricular rhythm without visible or regular p-waves for 30 seconds, whereas atrial flutter was defined by regular P waves at a rate of $250-350 / \mathrm{min}$.

\section{End Points}

The co-primary endpoints were time to and frequency of conversion to SR. The secondary endpoints were serious adverse (e.g. ventricular arrhythmia, symptomatic bradycardia, death) and adverse events (e.g. pain at the infusion site) during infusion, detection of AF or atrial flutter, hospital visits due to AF or atrial flutter, episodes with tachycardia lasting for at least one hour, and serum- and plasma-potassium during follow up.

\section{Statistics}

We assumed that 50\% of the patients would convert spontaneously to SR 4, 19 and postulated that potassium infusion would increase the rate of conversion to $75 \%$. With a power of $80 \%$ 
to detect this increase, at least $5^{8}$ patients were predicted to be required in both the $\mathrm{KCl}$ infusion and placebo groups to detect a significant treatment effect.

Continuous variables are presented with median and interquartile range (IQR), and categorical variables are presented as count and percentage. Statistical differences between any two groups were examined with Pearson's Chi-squared test for categorical variables and a 2-sample Wilcoxon rank-sum (Mann-Whitney) test applied for continuous variables.

Differences in time to conversion between groups were also assessed with Kaplan-Meier plots, a log-rank test, and Cox proportional hazard regressions. The assumption of proportional hazard was tested using Schoenfeld residuals as well as visual inspection of a $\log -\log$ plot.

The primary analysis was intention-to-treat and involved all randomized patients included. All patients who were randomized into either group were considered to be in the intentionto-treat sample ( $\mathrm{n}=113)$. From these, 5 patients were excluded from the placebo group and 11 patients were excluded from the $\mathrm{KCl}$ group (Figure 1). All other patients for whom the protocol was followed $(n=97)$ were included in a prespecified per-protocol analysis with the same endpoints as for the intention-to-treat analysis.

Statistical analyses were performed using Stata/SE version 14.2 (Stata Corporation, College Station, TX). A P value $\leq 0.05$ was considered statistically significant.

\section{Results}

\section{Study Participants}

Due to slower inclusion than expected, a total of 113 patients were randomized, of whom 53 were allocated to the placebo group and 60 to the $\mathrm{KCl}$ group (Figure 1). The vast majority of included patients presented exclusively with $\mathrm{AF}$ (>90\%), while 9 patients (8\%) had atrial flutter and 2 patients (1.8\%) alternated between $\mathrm{AF}$ and atrial flutter. Eleven patients received $\mathrm{KCl}$ at an infusion rate of $166 \mathrm{~mL} / \mathrm{h}(9.4 \mathrm{mmol} \mathrm{KCl} / \mathrm{h}), 19$ patients at a rate of 12 $\mathrm{mmol} \mathrm{KCl} / \mathrm{h}$, and 30 patients at a rate of $15 \mathrm{mmol} \mathrm{KCl} / \mathrm{h}$. Forty-eight patients in the placebo 
1 group and 49 patients in the $\mathrm{KCl}$ group completed the protocol. Baseline characteristics

2 (Table 1) were similar between these groups, apart from a higher percentage of men and a

3 higher median LVEDD in the $\mathrm{KCl}$ group. However, adjusting for these variables in Cox

4 regression models did not change our result to any significant degree.

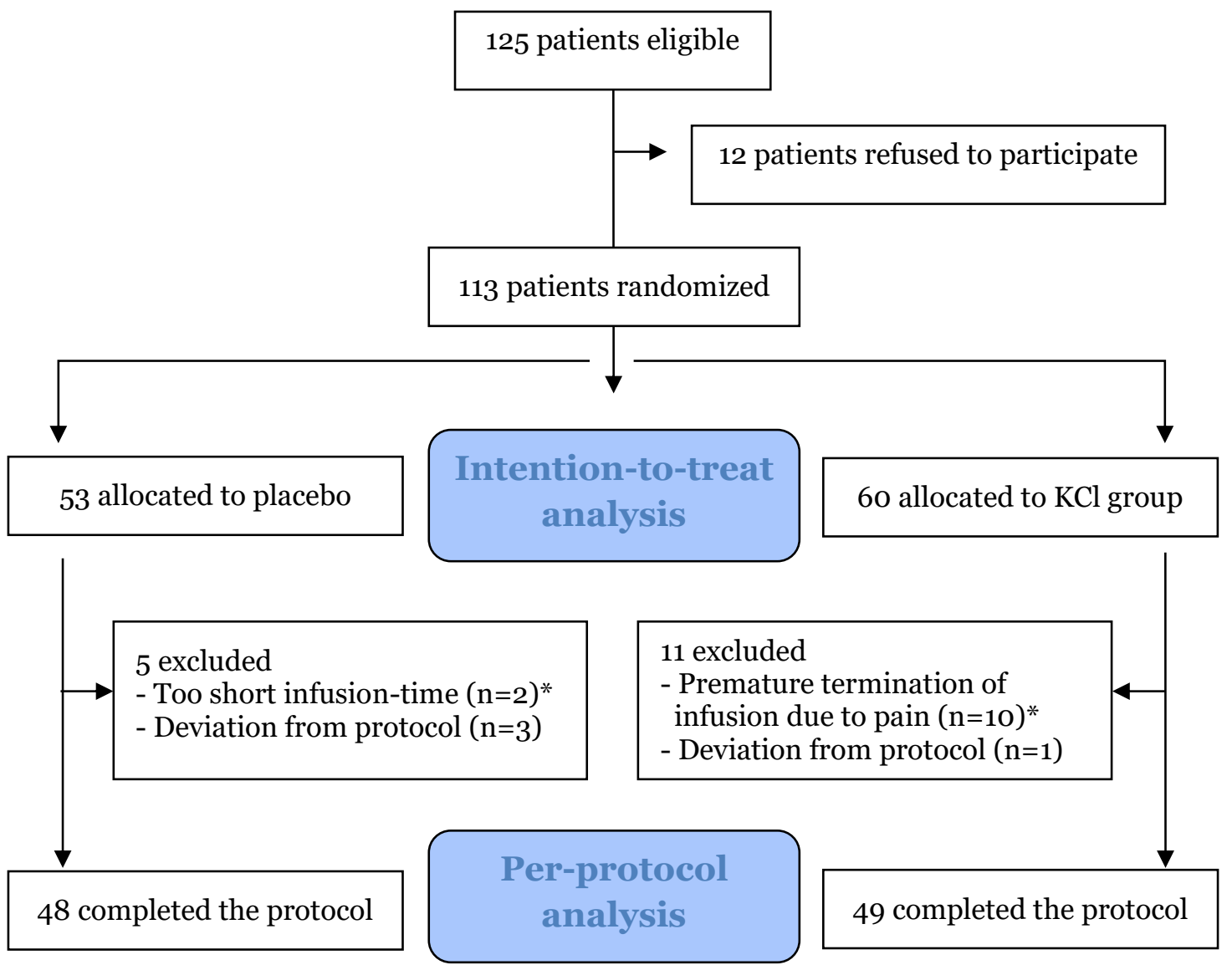

6 Figure 1. Study enrollment and randomization.

$7 \mathrm{KCl}$, potassium chloride. * Patients who had received fluid corresponding to $<10 \mathrm{mmol} \mathrm{KCl}$ in the

8 placebo group and $<10(1.25-6) \mathrm{mmol} \mathrm{KCl}$ in the $\mathrm{KCl}$ group.

9

Table 1. Baseline characteristics

$\begin{array}{lcc} & \text { Placebo (n=53) } & \text { KCl (n=6o) } \\ \text { Age, y } & 63(54-73) & 67(53-74) \\ \text { Sex, \% male } & 27(50.9 \%) & \mathbf{4 4}(\mathbf{7 3 . 3 \%})^{*} \\ \text { BMI, kg/m² } & 24.6(22.5-27.2) & 25.8(22.9-28.1) \\ \text { Duration of symptoms, hrs } & 2.75(1.8-5.0) & 4.2(2.0-12.5) \\ \text { Heart rate, bpm } & 128(116-143) & 131(111-148) \\ \text { Medical history } & & \\ \text { Atrial fibrillation } & 32(60.4 \%) & 39(65.0 \%) \\ \text { Kidney function (eGFR) } & & \\ -\quad \geq 60 \text { mL/min } & 44(83.0 \%) & 53(88.3 \%) \\ \text { Hypertension } & 19(35.9 \%) & 19(31.7 \%) \\ \text { Stroke/TIA } & 5(9.4 \%) & 2(3.3 \%)\end{array}$




\begin{tabular}{|c|c|c|}
\hline Coronary heart disease & $3(5.7 \%)$ & $7(11.7 \%)$ \\
\hline Previous ablation & $3(5.7 \%)$ & $5(8.3 \%)$ \\
\hline Diabetes mellitus & $2(3.8 \%)$ & $4(6.7 \%)$ \\
\hline Hypo-/hyperthyroidism & $3(5.7 \%)$ & $3(5 \%)$ \\
\hline \multicolumn{3}{|l|}{ Medications } \\
\hline ASA & $8(15.1 \%)$ & $12(20.0 \%)$ \\
\hline Warfarin & $5(9.4 \%)$ & $6(10 \%)$ \\
\hline NOAC & $12(22.6 \%)$ & $10(16.7 \%)$ \\
\hline Beta-blocker & $14(26.4 \%)$ & $20(33.3 \%)$ \\
\hline Digoxin & $1(1.9 \%)$ & o \\
\hline ACE-I or ARB & $15(28.9 \%)$ & $15(25 \%)$ \\
\hline Diuretic (loop or thiazide) & $9(17.0 \%)$ & $13(21.7 \%)$ \\
\hline Potassium-sparing diuretic & $1(1.9 \%)$ & $1(1.7 \%)$ \\
\hline CCB (non-dihydropyridines) & $6(11.3 \%)$ & $8(13.3 \%)$ \\
\hline CCB (dihydropyridines) & $1(1.9 \%)$ & $2(3.3 \%)$ \\
\hline Statin & $12(22.6 \%)$ & $13(21.7 \%)$ \\
\hline Levothyroxine & $2(3.8 \%)$ & $2(3.3 \%)$ \\
\hline \multicolumn{3}{|c|}{ Echocardiographic characteristics } \\
\hline LAA, $\mathrm{cm}^{2}, \mathrm{n}=107$ & $21(18-27)$ & $22.5(19-27)$ \\
\hline LVEDD, mm, n=108 & $47(44-52)$ & $50(47-54)^{*}$ \\
\hline LV mass, $g, n=107$ & $60.7(45.8-83.3)$ & $66.3(52.8-81.7)$ \\
\hline $\mathrm{LVEF} \geq 50 \%, \mathrm{n}=107$ & $51(98.1 \%)$ & $52(94.6 \%)$ \\
\hline Mitral regurgitation ${ }^{\dagger}, \mathrm{n}=108$ & $4(7.7 \%)$ & $7(12.5 \%)$ \\
\hline E/A-ratio, $n=89$ & $1.2(0.9-1.6)$ & $1.2(1.1-1.7)$ \\
\hline $\mathrm{E} / \mathrm{e}^{\prime}, \mathrm{n}=95$ & $7.9(6.0-10.1)$ & $7.7(5.8-10.5)$ \\
\hline SPAP, mmHg, n=59 & $30.5(26.5-36.0)$ & $26.5(25.0-30.0)$ \\
\hline \multicolumn{3}{|l|}{ Pre-infusion } \\
\hline $\mathrm{S}$-potassium, mmol/L & $4.1(3.8-4.2)$ & $4.1(3.9-4.2)$ \\
\hline $\mathrm{S}$-potassium $<3,5 \mathrm{mmol} / \mathrm{L}, \mathrm{n}=3$ & $3 \cdot 3(3 \cdot 3-3 \cdot 3)$ & $3.4(3 \cdot 3-3.4)$ \\
\hline P-potassium, mmol/L & $3.8(3.6-4.0)$ & $3.8(3.6-3.9)$ \\
\hline $\mathrm{P}$-potassium $<3,5 \mathrm{mmol} / \mathrm{L}, \mathrm{n}=11$ & $3 \cdot 3(3.3-3.4)$ & $3.4(3 \cdot 2-3 \cdot 4)$ \\
\hline Magnesium, mmol/L, $\mathrm{n}=111$ & $0.81(0.78-0.85)$ & $0.83(0.76-0.87)$ \\
\hline Creatinine, $\mu \mathrm{mol} / \mathrm{L}$ & $79(66-91)$ & $80(71-90)$ \\
\hline NT-proBNP, ng/L, n=97 & $262(80-1023)$ & $304(127-1023)$ \\
\hline Troponin $\mathrm{T}, \mathrm{ng} / \mathrm{L}, \mathrm{n}=111$ & $11(10-16)$ & $11(10-17)$ \\
\hline CRP, mg/L & $1(1-2)$ & $1(1-2)$ \\
\hline $\mathrm{TSH}, \mathrm{mIU} / \mathrm{L}, \mathrm{n}=100$ & $2.2(1.1-3.1)$ & $2.3(1.5-3.4)$ \\
\hline Free $\mathrm{T}_{4}, \mathrm{pmol} / \mathrm{L}, \mathrm{n}=100$ & $16(15-18)$ & $16(15-17)$ \\
\hline
\end{tabular}

1 Values are median and interquartile range or number and percentage.

$2+$ Moderate to severe. ${ }^{*} \mathrm{p}$ value $<0.05$

3 BMI: body mass index; TIA: transient ischemic attack; ASA: Acetylsalicylic acid; NOAC: Non-vitamin

$4 \quad \mathrm{~K}$ antagonist oral anticoagulant; ACE-I: angiotensin converting enzyme inhibitor; ARB: Angiotensin

5 receptor II blocker; CCB: calcium channel blocker; LAA: left atrial area; LVEDD: left ventricle end-

6 diastolic diameter; LV: left ventricle; LVEF: left ventricle ejection fraction; E/A: ratio between mitral

7 valve early and late diastolic inflow; E/e': ratio between early mitral inflow velocity and mitral annular

8 early diastolic velocity; SPAP: Systolic pulmonary artery pressure

$9 \quad$ Time to and frequency of conversion to SR 
1 There was no significant effect of $\mathrm{KCl}$ infusion on the co-primary endpoint of time to or

2 frequency of conversion to SR compared with placebo when all $\mathrm{KCl}$ patients were grouped

3 independent of infusion rate (Table 2 and 3).

Table 2. Outcomes by intention-to-treat analysis

\begin{tabular}{|c|c|c|c|c|c|}
\hline \multirow[t]{2}{*}{ Variables } & \multicolumn{2}{|c|}{ All participants } & \multirow{2}{*}{$\begin{array}{c}265 \mathrm{~mL} / \mathrm{h} \\
\mathrm{KCl} \\
(15 \mathrm{mmol} / \mathrm{h}) \\
(\mathrm{n}=\mathbf{3 0})\end{array}$} & \multicolumn{2}{|c|}{ All KCl } \\
\hline & $\begin{array}{c}\text { Placebo } \\
(n=53)\end{array}$ & $\begin{array}{c}\text { KCl } \\
(n=60)\end{array}$ & & $\begin{array}{c}\Delta P \text {-potassium } \\
\text { below median } \\
\text { p- } \\
\text { potassium } / \mathbf{h} \\
(\mathbf{n}=\mathbf{2 8})\end{array}$ & $\begin{array}{c}\Delta P-p o t a s s i u m \\
\text { above median } \\
\text { p- } \\
\text { potassium } / \mathbf{h} \\
(n=28)\end{array}$ \\
\hline Time to conversion, $\mathrm{h}$ & $\begin{array}{c}6.8 \\
(1.6-15 \cdot 5)\end{array}$ & $\begin{array}{c}5 \cdot 5 \\
(2.3-12.1)\end{array}$ & $\begin{array}{c}5 \cdot 3 \\
(1.8-10.9)\end{array}$ & $\begin{array}{c}8.1 \\
(3.0-13.2)\end{array}$ & $\begin{array}{c}5 \cdot 4 \\
(2.2-11.7)\end{array}$ \\
\hline $\begin{array}{l}\text { Number of patients } \\
\text { who converted to SR }\end{array}$ & $\begin{array}{c}28 \\
(52.8 \%)\end{array}$ & $\begin{array}{c}34 \\
(56.7 \%)\end{array}$ & $\begin{array}{c}20 \\
(66.7 \%)\end{array}$ & $\begin{array}{c}10 \\
(35 \cdot 7 \%)\end{array}$ & $\begin{array}{c}23^{* *} \\
(\mathbf{8 2 . 1 \% )}\end{array}$ \\
\hline Infusion time, $\mathrm{h}$ & $\begin{array}{c}13.0 \\
(4.7-17.2)\end{array}$ & $\begin{array}{c}9.6 \\
(2.8-16.0)\end{array}$ & $\begin{array}{c}6.0 \\
(2.2-13.5)\end{array}$ & $\begin{array}{c}13.8 \\
(9.8-22.7)\end{array}$ & $\begin{array}{c}5.6^{*} \\
(2.2-10.7)\end{array}$ \\
\hline $\begin{array}{l}\text { Total potassium } \\
\text { infused, mmol }\end{array}$ & o & $\begin{array}{c}120.1 \\
(34.1-209.4)\end{array}$ & $\begin{array}{c}90.0 \\
(32.5-202.5)\end{array}$ & $\begin{array}{c}169.5 \\
(107.8-255)\end{array}$ & $\begin{array}{c}82 \\
(33.1-143.8)\end{array}$ \\
\hline Pain from the infusion & o & $\begin{array}{l}12^{* *} \\
(20 \%)\end{array}$ & $\begin{array}{l}\mathbf{9}^{* * *} \\
(\mathbf{3 0} \%)\end{array}$ & $\begin{array}{c}2^{*} \\
(7.1 \%)\end{array}$ & $\begin{array}{c}6^{* * *} \\
(21.4 \%)\end{array}$ \\
\hline S-potassium, mmol/L $\mathrm{L}^{\dagger}$ & $\begin{array}{c}4.0 \\
(3.8-4.2)\end{array}$ & $\begin{array}{c}4.7 \\
(4 \cdot 3-5 \cdot 1)\end{array}$ & $\begin{array}{c}4.6 \\
(4.2-5.1)\end{array}$ & $\begin{array}{c}4.7 \\
(4.4-5.1)\end{array}$ & $\begin{array}{c}4.6 \\
(4.2-5.1)\end{array}$ \\
\hline P-potassium, mmol/L $\mathrm{L}^{\dagger}$ & $\begin{array}{c}3.8 \\
(3.6-3.8)\end{array}$ & $\begin{array}{c}4.3 \\
(4.1-4 \cdot 6)\end{array}$ & $\begin{array}{c}4.4 \\
(4.0-4 \cdot 7)\end{array}$ & $\begin{array}{c}4 \cdot 3 \\
(4 \cdot 2-4.6)\end{array}$ & $\begin{array}{c}4.4 \\
(4.0-4 \cdot 7)\end{array}$ \\
\hline
\end{tabular}

$4 \quad$ Values are median and interquartile range or number and percentage.

$5 \quad{ }^{*} \mathrm{P}$ value $<0.05 ;{ }^{* *} \mathrm{P}$ value $<0.01 ;{ }^{* * *} \mathrm{P}$ value $<0.001$

6 † After stopping the infusion (post-infusion).

$7 \quad$ All treatment groups were statistically compared with the placebo group.

Table 3. Outcomes by per-protocol analysis

\begin{tabular}{|c|c|c|c|c|c|}
\hline \multirow[t]{2}{*}{ Variables } & \multicolumn{2}{|c|}{ All participants } & \multirow{2}{*}{$\begin{array}{c}265 \mathrm{ml} / \mathrm{h} \\
\mathrm{KCl} \\
(15 \mathrm{mmol} / \mathrm{h}) \\
(\mathrm{n}=\mathbf{2 2})\end{array}$} & \multicolumn{2}{|c|}{ All KCl } \\
\hline & $\begin{array}{l}\text { Placebo } \\
(n=48)\end{array}$ & $\begin{array}{c}\mathrm{KCl} \\
(n=49)\end{array}$ & & $\begin{array}{c}\Delta P-p o t a s s i u m \\
\text { below median } \\
\text { p- } \\
\text { potassium } / \mathbf{h} \\
(\mathbf{n}=25)\end{array}$ & $\begin{array}{c}\Delta P- \\
\text { potassium } \\
\text { above } \\
\text { median p- } \\
\text { potassium } / \mathbf{h} \\
(n=24)\end{array}$ \\
\hline Time to conversion, $\mathrm{h}$ & $\begin{array}{c}7 \\
(2-15.6)\end{array}$ & $\begin{array}{c}5 \cdot 7 \\
(2.3-12.4)\end{array}$ & $\begin{array}{c}5 \cdot 3 \\
(2.2-12.1)\end{array}$ & $\begin{array}{c}8.1 \\
(3.0-13.2)\end{array}$ & $\begin{array}{c}5.4 \\
(2.2-11.7)\end{array}$ \\
\hline $\begin{array}{l}\text { Number of patients } \\
\text { who converted to SR }\end{array}$ & $\begin{array}{c}25 \\
(52.1 \%)\end{array}$ & $\begin{array}{c}32 \\
(65.3 \%)\end{array}$ & $\begin{array}{c}18 * \\
(81.8 \%)\end{array}$ & $\begin{array}{c}10 \\
(40.0 \%)\end{array}$ & $\begin{array}{l}22^{* *} \\
(91.7)\end{array}$ \\
\hline Infusion-time, $\mathrm{h}$ & $\begin{array}{c}13 \cdot 4 \\
(5.8-17.6)\end{array}$ & $\begin{array}{c}12.1 \\
(3 \cdot 9-17 \cdot 9)\end{array}$ & $\begin{array}{c}6.8 \\
(2.2-14.2)\end{array}$ & $\begin{array}{c}14 \\
(11.8-23.0)\end{array}$ & $\begin{array}{c}5 \cdot 7^{*} \\
(2.2-12.9)\end{array}$ \\
\hline $\begin{array}{l}\text { Total potassium } \\
\text { infused, mmol }\end{array}$ & o & $\begin{array}{c}141.0 \\
(37.1-222.7)\end{array}$ & $\begin{array}{c}102.6 \\
(33.7-213.7)\end{array}$ & $\begin{array}{c}180.0 \\
(119.0-242.0)\end{array}$ & $\begin{array}{c}80.5 \\
(33.1-192.9)\end{array}$ \\
\hline Pain from the infusion & o & $\begin{array}{c}2 \\
(4.1 \%)\end{array}$ & $\begin{array}{c}2^{*} \\
(9.1 \%)\end{array}$ & $\begin{array}{c}1 \\
(4.0 \%)\end{array}$ & $\begin{array}{c}1 \\
(4.2 \%)\end{array}$ \\
\hline $\mathrm{S}$-potassium, mmol/L $\mathrm{L}^{\dagger}$ & $\begin{array}{c}4.0 \\
(3.8-4.2)\end{array}$ & $\begin{array}{c}4.7 \\
(4 \cdot 3-5 \cdot 1)\end{array}$ & $\begin{array}{c}4.6 \\
(4.2-5.1)\end{array}$ & $\begin{array}{c}4.7 \\
(4.4-5.1)\end{array}$ & $\begin{array}{c}4.6 \\
(4.2-5.1)\end{array}$ \\
\hline $\mathrm{P}$-potassium, mmol/L $\mathrm{L}^{\dagger}$ & $\begin{array}{c}3.8 \\
(3.6-3.8)\end{array}$ & $\begin{array}{c}4.3 \\
(4.1-4.6)\end{array}$ & $\begin{array}{c}4.4 \\
(4.0-4.7)\end{array}$ & $\begin{array}{c}4.3 \\
(4.2-4.6)\end{array}$ & $\begin{array}{c}4.4 \\
(4.0-4 \cdot 7)\end{array}$ \\
\hline
\end{tabular}


1 Values are median and interquartile range or number and percentage.

$2 \quad{ }^{*} \mathrm{P}$ value $<0.05 ;{ }^{* *} \mathrm{P}$ value $<0.01 ;{ }^{* * *} \mathrm{P}$ value $<0.001$

$3+$ After stopping the infusion (post-infusion).

4 All treatment groups were statistically compared with the placebo group.

5 A Kaplan-Meier plot showed only a trend toward increased number of patients who

6 converted to SR, which was non-significant by logrank test $(P=0.29$, Figure 2a), and Cox

7 regression showed a hazard ratio (HR) of $1.20(\mathrm{CI} 0.72-1.98, P=0.72)$ for $\mathrm{KCl}$ infusion

8 compared with placebo. When analyzing the $\mathrm{KCl}$ group receiving the fastest infusion $\mathrm{KCl}$ rate

9 of $15 \mathrm{mmol} / \mathrm{h}$, there was still no significant difference in conversion rate nor in time to conversion in the intention-to-treat analysis compared with placebo. However, when comparison was done in patients without a history of AF, significantly more patients in the $\mathrm{KCl}$ group converted to SR compared with the placebo group (85.7 vs $52.4 \% ; P=0.019$;

Supplementary Table 1a) independently of infusion rate of $\mathrm{KCl}$.

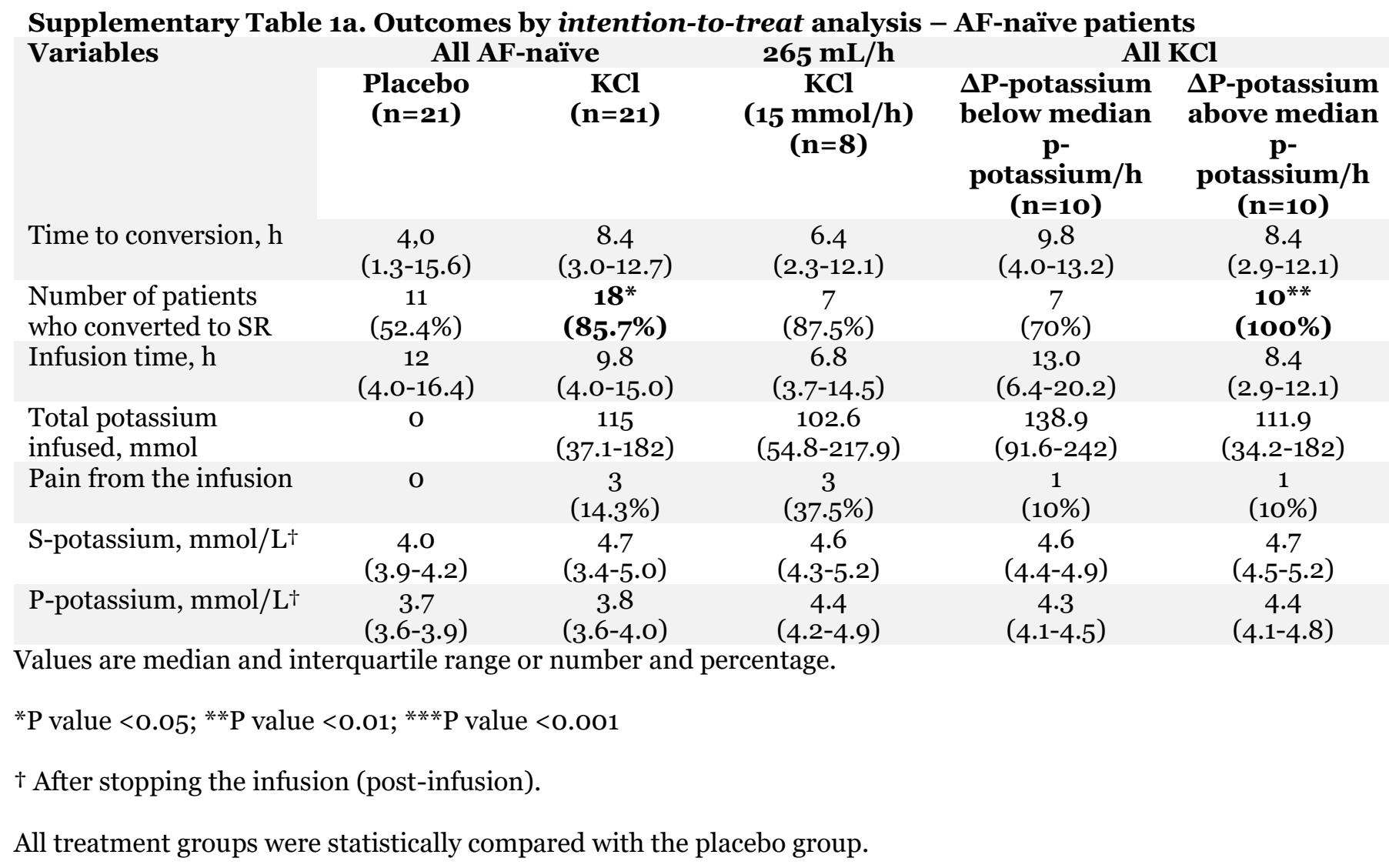




\begin{tabular}{|c|c|c|c|c|c|}
\hline \multirow[t]{2}{*}{$\begin{array}{l}\text { Suppleme } \\
\text { Variables }\end{array}$} & \multicolumn{2}{|c|}{ All not AF-naïve } & \multirow{2}{*}{$\begin{array}{c}265 \mathrm{~mL} / \mathrm{h} \\
\mathrm{KCl} \\
(15 \mathrm{mmol} / \mathrm{h}) \\
(\mathrm{n}=\mathbf{2 2})\end{array}$} & \multicolumn{2}{|c|}{$\begin{array}{l}\text { Ith history } \\
\text { All KCl }\end{array}$} \\
\hline & $\begin{array}{c}\text { Placebo } \\
(n=32)\end{array}$ & $\begin{array}{c}\text { KCl } \\
(n=39)\end{array}$ & & $\begin{array}{c}\Delta P \text {-potassium } \\
\text { below median } \\
\text { p- } \\
\text { potassium } / \mathbf{h} \\
(\mathbf{n}=\mathbf{1 8})\end{array}$ & $\begin{array}{c}\Delta \text { P-potassium } \\
\text { above median } \\
\text { p- } \\
\text { potassium } / \mathbf{h} \\
(\mathbf{n}=\mathbf{1 8})\end{array}$ \\
\hline Time to conversion, $\mathrm{h}$ & $\begin{array}{c}7 \\
(1.7-15.4)\end{array}$ & $\begin{array}{c}3 \cdot 7 \\
(1.7-7 \cdot 7)\end{array}$ & $\begin{array}{c}5.2 \\
(1.5-9.7)\end{array}$ & $\begin{array}{c}2.3 \\
(1.0-13.5)\end{array}$ & $\begin{array}{c}5 \cdot 2 \\
(1.8-5.8)\end{array}$ \\
\hline $\begin{array}{l}\text { Number of patients } \\
\text { who converted to SR }\end{array}$ & $\begin{array}{c}17 \\
(53.1 \%)\end{array}$ & $\begin{array}{c}16 \\
(41.0 \%)\end{array}$ & $\begin{array}{c}13 \\
(59.1 \%)\end{array}$ & $\begin{array}{c}3 \\
(16.7 \%)\end{array}$ & $\begin{array}{c}13 \\
(72.2 \%)\end{array}$ \\
\hline Infusion time, $\mathrm{h}$ & $\begin{array}{c}13 \cdot 3 \\
(4 \cdot 7-17.6)\end{array}$ & $\begin{array}{c}9.5 \\
(2.1-17.1)\end{array}$ & $\begin{array}{c}5.6 \\
(2.1-13.5)\end{array}$ & $\begin{array}{c}15 \cdot 5 \\
(11.8-23 \cdot 3)\end{array}$ & $\begin{array}{c}5.5^{*} \\
(1.9-9.5)\end{array}$ \\
\hline $\begin{array}{l}\text { Total potassium } \\
\text { infused, mmol }\end{array}$ & o & $\begin{array}{c}131.5 \\
(31.3-213.8)\end{array}$ & $\begin{array}{c}83.6 \\
\left(31.3^{-202.5)}\right.\end{array}$ & $\begin{array}{c}203.8 \\
(141-268)\end{array}$ & $\begin{array}{c}79 \\
(22.8-142.5)\end{array}$ \\
\hline Pain from the infusion & o & $\begin{array}{c}9 \\
(23.1 \%)\end{array}$ & $\begin{array}{c}6 \\
(27.3 \%)\end{array}$ & $\begin{array}{c}1 \\
(5.6 \%)\end{array}$ & $\begin{array}{c}5 \\
(27.8 \%)\end{array}$ \\
\hline S-potassium, mmol/L $\mathrm{L}^{\dagger}$ & $\begin{array}{c}4.0 \\
(3.7-4.3)\end{array}$ & $\begin{array}{c}4.7 \\
(4 \cdot 3-5 \cdot 1)\end{array}$ & $\begin{array}{c}4.5 \\
(4.2-5.1)\end{array}$ & $\begin{array}{c}4.8 \\
(4.4-5.1)\end{array}$ & $\begin{array}{c}4.4 \\
(4.2-5.1)\end{array}$ \\
\hline P-potassium, mmol/L $\mathrm{L}^{\dagger}$ & $\begin{array}{c}3.8 \\
(3.6-4.0)\end{array}$ & $\begin{array}{c}3.8 \\
(3.7-3.9)\end{array}$ & $\begin{array}{c}4.3 \\
(3.9-4.7)\end{array}$ & $\begin{array}{c}4.3 \\
(4.2-4.6)\end{array}$ & $\begin{array}{c}4.3 \\
(4.0-4 \cdot 7)\end{array}$ \\
\hline
\end{tabular}

Twelve patients in the $\mathrm{KCl}$ group experienced pain at the infusion site (20\%), and most of these were allocated to receiving $\mathrm{KCl}$ at the fastest infusion rate of $15 \mathrm{mmol} / \mathrm{h}$. In 10 of these patients, the $\mathrm{KCl}$ infusion had to be prematurely terminated due to the pain (Table 2). After excluding these patients, the per-protocol analysis showed that significantly more patients converted to SR in the $\mathrm{KCl} 15 \mathrm{mmol} / \mathrm{h}$ group compared with the placebo group (82\% vs 53\%. $P=0.018$, Table 3). Significant differences between the $\mathrm{KCl} 15 \mathrm{mmol} / \mathrm{h}$ and placebo groups were also observed when tested with a Kaplan Meier plot (logrank $P=0.02)$ and Cox regression (HR 2.26, CI 1.09-3.71, $P=0.024$, Figure 2b). Again, when comparison was done in patients without a history of $\mathrm{AF}$, significantly more patients in the $\mathrm{KCl}$ group converted to $\mathrm{SR}$ compared with the placebo group ( $85 \%$ vs $47.4 \% ; P=0.013$; Supplementary Table 2a) independently of infusion rate of $\mathrm{KCl}$.

Supplementary Table 2a. Outcomes by per protocol analysis - AF-naïve patients Variables

All AF-naive Placebo $(n=19)$ $\mathrm{KCl}$ $(\mathbf{n}=\mathbf{2 0})$ $265 \mathrm{~mL} / \mathrm{h}$ $\mathrm{KCl}$ (15 mmol/h)
All KCl $\Delta \mathbf{P}$-potassium $\quad \Delta \mathbf{P}$-potassium below median above median 


\begin{tabular}{|c|c|c|c|c|c|}
\hline & & & $(n=7)$ & 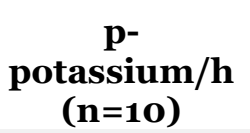 & 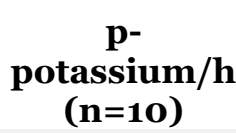 \\
\hline Time to conversion, $\mathrm{h}$ & $\begin{array}{c}4 \\
(2.2-15.6)\end{array}$ & $\begin{array}{c}9.6 \\
(4.0-12.7)\end{array}$ & $\begin{array}{c}6.8 \\
(5.1-12.1)\end{array}$ & $\begin{array}{c}9.8 \\
(4.0-13.2)\end{array}$ & $\begin{array}{c}8.4 \\
(2.9-12.1)\end{array}$ \\
\hline $\begin{array}{l}\text { Number of patients } \\
\text { who converted to SR }\end{array}$ & $\begin{array}{c}9 \\
(47.4 \%)\end{array}$ & $\begin{array}{l}17^{*} \\
(85 \%)\end{array}$ & $\begin{array}{c}6 \\
(85.7 \%)\end{array}$ & $\begin{array}{c}7 \\
(70 \%)\end{array}$ & $\begin{array}{c}10^{* *} \\
(100 \%)\end{array}$ \\
\hline Infusion time, $\mathrm{h}$ & $\begin{array}{c}12 \\
(4 \cdot 0-17 \cdot 3)\end{array}$ & $\begin{array}{c}10.7 \\
(4.5-16.0)\end{array}$ & $\begin{array}{c}7 \cdot 3 \\
(5.1-16.9)\end{array}$ & $\begin{array}{c}13.0 \\
(6.4-20.2)\end{array}$ & $\begin{array}{c}8.4 \\
(2.9-12.1)\end{array}$ \\
\hline $\begin{array}{l}\text { Total potassium } \\
\text { infused, mmol }\end{array}$ & 0 & $\begin{array}{c}117.0 \\
(56.4-202.3)\end{array}$ & $\begin{array}{c}108.8 \\
(75.8-253.8)\end{array}$ & $\begin{array}{c}138.9 \\
(91.6-242)\end{array}$ & $\begin{array}{c}111.9 \\
(34.2-182)\end{array}$ \\
\hline Pain from the infusion & o & $\begin{array}{c}2 \\
(10 \%)\end{array}$ & $\begin{array}{c}2 \\
(28.6 \%)\end{array}$ & $\begin{array}{c}1 \\
(10 \%)\end{array}$ & $\begin{array}{c}1 \\
(10 \%)\end{array}$ \\
\hline S-potassium, mmol/L† & $\begin{array}{c}4.1 \\
(3.9-4.2)\end{array}$ & $\begin{array}{c}4.7 \\
(4.4-5.0)\end{array}$ & $\begin{array}{c}4.6 \\
(4.3-5.2)\end{array}$ & $\begin{array}{c}4.6 \\
(4.4-4.9)\end{array}$ & $\begin{array}{c}4.7 \\
(4.5-5.2)\end{array}$ \\
\hline P-potassium, mmol/L $\mathrm{L}^{\dagger}$ & $\begin{array}{c}3.8 \\
(3.5-3.9)\end{array}$ & $\begin{array}{c}4 \cdot 4 \\
(4.1-4.6)\end{array}$ & $\begin{array}{c}4.4 \\
(4.2-4.9)\end{array}$ & $\begin{array}{c}4 \cdot 3 \\
(4.1-4.5)\end{array}$ & $\begin{array}{c}4.4 \\
(4.1-4.8)\end{array}$ \\
\hline
\end{tabular}

1 Values are median and interquartile range or number and percentage.

$2 \quad{ }^{*}$ P value $<0.05 ;{ }^{* *} \mathrm{P}$ value $<0.01 ;{ }^{* * *} \mathrm{P}$ value $<0.001$

3 t After stopping the infusion (post-infusion).

4 All treatment groups were statistically compared with the placebo group.

\begin{tabular}{|c|c|c|c|c|c|}
\hline \multirow[t]{2}{*}{$\begin{array}{l}\text { Suppleme } \\
\text { Variables }\end{array}$} & $\begin{array}{l}\text { Dutcom } \\
\text { All no }\end{array}$ & $\begin{array}{l}\text { y per prot } \\
\text { F-naïve }\end{array}$ & \multirow{2}{*}{$\begin{array}{c}265 \mathrm{~mL} / \mathrm{h} \\
\mathrm{KCl} \\
(15 \mathrm{mmol} / \mathrm{h}) \\
(\mathrm{n}=15)\end{array}$} & \multicolumn{2}{|c|}{ All KCl } \\
\hline & $\begin{array}{l}\text { Placebo } \\
(\mathbf{n}=29)\end{array}$ & $\begin{array}{c}\text { KCl } \\
(n=29)\end{array}$ & & $\begin{array}{c}\Delta \mathbf{P}-\text { potassium } \\
\text { below median } \\
\text { p- } \\
\text { potassium } / \mathbf{h} \\
(\mathbf{n}=\mathbf{1 8})\end{array}$ & $\begin{array}{l}\Delta P \text {-potassium } \\
\text { above median } \\
\text { p- } \\
\text { potassium } / \mathbf{h} \\
(\mathbf{n}=\mathbf{1 8})\end{array}$ \\
\hline Time to conversion, $\mathrm{h}$ & $\begin{array}{c}7 \cdot 4 \\
(1.8-17 \cdot 4)\end{array}$ & $\begin{array}{c}2.3 \\
(1.5-9.7)\end{array}$ & $\begin{array}{c}3 \cdot 7 \\
(1.4-11.6)\end{array}$ & $\begin{array}{c}2.3 \\
(1.0-13.5)\end{array}$ & $\begin{array}{c}3.4 \\
(1.7-7 \cdot 7)\end{array}$ \\
\hline $\begin{array}{l}\text { Number of patients } \\
\text { who converted to SR }\end{array}$ & $\begin{array}{c}16 \\
(55.2 \%)\end{array}$ & $\begin{array}{c}15 \\
(51.7 \%)\end{array}$ & $\begin{array}{c}12 \\
(80.0 \%)\end{array}$ & $3(20 \%)$ & $\begin{array}{c}12 * \\
(85.7 \%)\end{array}$ \\
\hline Infusion time, $\mathrm{h}$ & $\begin{array}{c}14 \\
(7.0-17.9)\end{array}$ & $\begin{array}{c}13.3 \\
(2.3-21.2)\end{array}$ & $\begin{array}{c}5.6 \\
\left(1.5^{-14.3}\right)\end{array}$ & $\begin{array}{c}17.1 \\
(12.4-24)\end{array}$ & $\begin{array}{c}5 \cdot 4 \\
(1.8-13.6)\end{array}$ \\
\hline $\begin{array}{l}\text { Total potassium } \\
\text { infused, mmol }\end{array}$ & 0 & $\begin{array}{c}149 \\
(33.8-225.5)\end{array}$ & $\begin{array}{c}83.5 \\
(22.5-213.8)\end{array}$ & $\begin{array}{c}205 \\
(141-268)\end{array}$ & $\begin{array}{c}65.7 \\
(22.5-203.8)\end{array}$ \\
\hline Pain from the infusion & o & o & 0 & 0 & o \\
\hline S-potassium, mmol/ $\mathrm{L}^{\dagger}$ & $\begin{array}{c}4.0 \\
(3.7-4.2)\end{array}$ & $\begin{array}{c}4.7 \\
(4.2-5.1)\end{array}$ & $\begin{array}{c}4.5 \\
(4.2-5.1)\end{array}$ & $\begin{array}{c}4.8 \\
(4.5-5.1)\end{array}$ & $\begin{array}{c}4 \cdot 4 \\
(4.2-4 \cdot 7)\end{array}$ \\
\hline P-potassium, mmol/L $\mathrm{L}^{\dagger}$ & $\begin{array}{c}3.8 \\
(3.6-4.0)\end{array}$ & $\begin{array}{c}4.3 \\
(4.0-4.7)\end{array}$ & $\begin{array}{c}4 \cdot 3 \\
(3 \cdot 9-4 \cdot 7)\end{array}$ & $\begin{array}{c}4 \cdot 3 \\
(4.2-4.6)\end{array}$ & $\begin{array}{c}4.3 \\
(4.0-4.7)\end{array}$ \\
\hline
\end{tabular}

6 Values are median and interquartile range or number and percentage.

$7 \quad{ }^{*} \mathrm{P}$ value $<0.05 ;{ }^{* *} \mathrm{P}$ value $<0.01 ;{ }^{* * *} \mathrm{P}$ value $<0.001$

8 t After stopping the infusion (post-infusion).

9 All treatment groups were statistically compared with the placebo group. 

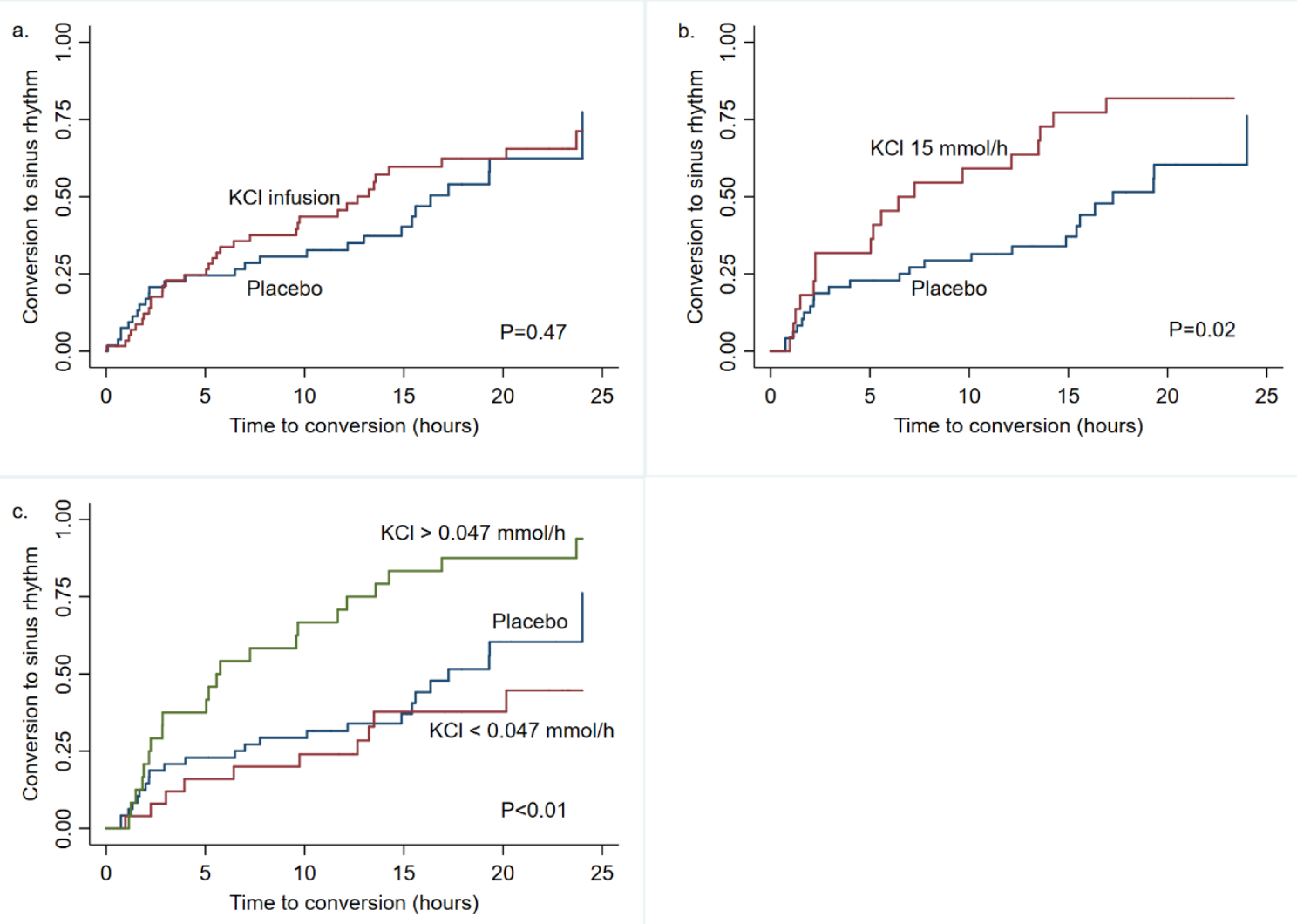

2 Figure 2. Kaplan - Meier curve for time to conversion and conversion to sinus rhythm (per protocol analysis). All patients who received $\mathrm{KCl}$ infusion $(\mathrm{n}=49)$ versus placebo $(\mathrm{n}=48)$ (a). Patients in the 15 $\mathrm{mmol} / \mathrm{h}$ group $(\mathrm{n}=22)$ versus placebo $(\mathrm{n}=48)(\mathbf{b})$. All patients who received $\mathrm{KCl}$ with a median plasma potassium change per hour above $(n=24)$ and below $(n=25)$ median $(0.047 \mathrm{mmol} / \mathrm{h})$ versus placebo $(\mathrm{n}=48)(\mathrm{c})$.

7 Of note, KCl-infused patients who achieved an above-median hourly increase in plasma-

8 potassium ( $>0.047 \mathrm{mmol} / \mathrm{h}$ ) exhibited a significantly higher conversion rate, but no difference in time to conversion compared with placebo (Table 2 and 3 and Supplementary Table 1a and 2a). Kaplan Meier plots also showed a significant difference between this abovemedian group compared with both the placebo group (logrank $P=0.002)$ and $\mathrm{KCl}$ patients with below-median change in plasma-potassium (logrank $P<0.001)$. There was no difference between the below-median group and placebo (logrank $P=0.09$, Figure 2c). Cox regression showed an HR of 2.40 (CI 1.36-4.21, $P=0.002$ ) when comparing above median change with placebo, and $\mathrm{HR}=4.41(\mathrm{CI} 2.07-9.40, P<0.001)$ compared with below median change. 
1 The group which received $\mathrm{KCl}$ at a rate of $15 \mathrm{mmol} / \mathrm{h}$ exhibited a greater increase in plasmapotassium (0.07 [0.04-0.18] mmol/L/h) than patients who received $9.4 \mathrm{mmol} / \mathrm{h}(0.04$ [0.03$0.05] \mathrm{mmol} / \mathrm{L} / \mathrm{h} ; P=0.015)$ and $12 \mathrm{mmol} / \mathrm{h}(0.04$ [0.03-0.06]; $P=0.043)$. Patients that converted to SR had a higher change in both serum- and plasma-potassium per hour than those that did not convert to sinus rhythm (0.08 [0.04-0.18] and 0.07 [0.03-0.18] mmol/L vs. 0.05 [0.02-0.06] and $0.04[0.03-0.05] \mathrm{mmol} / \mathrm{L}$, respectively, $P<0.01)$.

\section{When analyzing whether lower baseline plasma-potassium levels yield higher probability for} conversion to $\mathrm{SR}$ after $\mathrm{KCl}$ infusion, we found that in the $\mathrm{KCl}$ group receiving the highest infusion rate of $15 \mathrm{mmol} / \mathrm{h}$, all patients with plasma-potassium at baseline $<3.8 \mathrm{mmol} / \mathrm{L}$ $(n=13)$ converted to SR, whereas 70\% with plasma-potassium between 3.8-4.0 mmol/L $(n=17)$ converted to SR.

\section{$\underline{\text { Atrial flutter }}$}

For the small subgroup of patients with atrial flutter, only 2 of 9 patients (22\%) converted to SR, and both of these were in the placebo group. However, these findings should be interpreted cautiously due to the small number of atrial flutter patients included in this trial.

\section{$\underline{\text { Administration of } \mathrm{MgSO}_{4}}$}

A total of 14 (23.3\%) patients in the $\mathrm{KCl}$ group were also administered $\mathrm{MgSO}_{4} ; 4$ in the 9.4 $\mathrm{mmol} / \mathrm{h}$ group, 1 in the $12 \mathrm{mmol} / \mathrm{h}$ group, and 9 in the $15 \mathrm{mmol} / \mathrm{h}$ group. There was no significant difference $(P=0.26)$ between this subset of patients and those receiving only $\mathrm{KCl}$ regarding conversion to SR during the infusion.

\section{$\underline{\text { Heart rate-reducing medication }}$}

Seventy percent of the patients in the $\mathrm{KCl}$ group and $91 \%$ of the patients in the placebo group received heart rate-reducing medication. In the $\mathrm{KCl}$ group, $62 \%$ of the patients were treated with metoprolol, $5 \%$ with verapamil, and $3 \%$ with digoxin, respectively vs. $79 \%, 2 \%$, and $9 \%$, respectively, in the placebo group. Among the patients who converted to SR, $68 \%$ were 
1 treated with metoprolol, 6\% with verapamil, and 6\% with digoxin, respectively, in the $\mathrm{KCl}$

2 group vs. $82 \%, 0 \%$, and $11 \%$, respectively, in the placebo group.

\section{$3 \quad$ Follow-up}

4 At the follow-up consultation after discharge, most patients in both groups had SR at rest and

5 during the 3 days with ambulatory ECG recording (Table 4). No patients had atrial flutter.

6 Although numerically there was a higher proportion of patients in the placebo group than in

7 the $\mathrm{KCl}$ group who exhibited $\mathrm{AF}$ at rest or during ambulatory ECG registration, and these

8 patients had a lower number of contacts with the hospital for AF, these differences were not

9 significant.

\section{Table 4. Post-discharge follow-up}

Time of follow-up after discharge (months), $\mathrm{n}=102$

Heart rate, bpm

ECG at rest, $\mathrm{n}=102^{*}$

- Sinus rhythm

- Atrial fibrillation

- Atrial flutter

Ambulatory ECG, $\mathrm{n}=93^{\dagger}$

- Sinus rhythm

- Atrial fibrillation

- Atrial flutter

Number of contacts with Diakonhjemmet Hospital for atrial fibrillation/atrial flutter after discharge, $\mathrm{n}=102^{*}$

- No contacts

- One or more times

Total number of ECG-documented atrial fibrillation/atrial flutter, $\mathrm{n}=113$

History of palpitations during $\geq 1$ hour, $\mathrm{n}=102^{*}$

Serum-potassium, $\mathrm{mmol} / \mathrm{L}, \mathrm{n}=102^{*}$

Plasma-potassium, $\mathrm{mmol} / \mathrm{L}, \mathrm{n}=101^{\ddagger}$

Difference between serum- and plasma-potassium, $\mathrm{mmol} / \mathrm{L}, \mathrm{n}=101^{\ddagger}$

${ }^{*}$ Eleven patients did not attend the follow-up consultation.

Placebo $(n=48)$

$3.8(3.3-4.8)$

$65(57-75)$

$43(89.6 \%)$

$4(8.3 \%)$

o

$37(86.0 \%)$
$6(14.0 \%)$
0

$48(96.0 \%)$

$2(4.0 \%)$

$\mathrm{O}$

$\mathrm{KCl}(\mathbf{n}=\mathbf{5 4})$

$3.9(3.3-4.5)$

$65(57-76)$

$49(92.6 \%)$

$3(5.5 \%)$

0

36 (67.9.0\%)

$49(81.7 \%)$

17 (32.1\%)

$11(18.3 \%)$

25 (47.2\%)

$15(25.0 \%)$

$17(35.4 \%)$

$12(22.2 \%)$

$4.4(4.2-4.6)$

$4.3(4.1-4.4)$

$4.1(3.9-4.3)$

$4.0(3.9-4.2)$

$0.3(0.2-0.3)$

$0.3(0.2-0.4)$

† Eleven patients did not attend the follow-up consultation, and nine patients did not receive

ambulatory ECG monitoring since they exhibited atrial fibrillation in an ECG performed at rest.

${ }^{*}$ Eleven patients did not attend the follow-up consultation. In one patient plasma-potassium was not measured.

\section{Discussion}

This is the first study to investigate the effects of $\mathrm{KCl}$ infusion in patients with ROAF or atrial flutter. Our results did not demonstrate any significant effect of potassium infusion on the 
primary endpoint, time to and frequency of conversion to SR. However, the per protocol analysis indicates that an increase in circulating potassium may increase the likelihood of conversion of ROAF to SR if the patient does not experience pain at the infusion site but only if the hourly increase is sufficiently high.

Previous work has shown that roughly 50\% of patients with ROAF convert spontaneously to SR within 48 hours 4 , 19, well in accordance with the results in the placebo group in our study. However, few patients with atrial flutter converted to SR indicating that this arrhythmia may be less likely to spontaneously convert to SR and be less affected by potassium correction. Of note, the number of patients in our study who converted to SR after $\mathrm{KCl}$ infusion was the same as for the placebo-treated patients. However, in a post-hoc analysis, we found that an increase in circulating potassium in our patients with potassium levels in the lower normal range may promote conversion to SR if the speed of potassium increase is sufficiently high, independent of the total amount infused. In fact, the median total amount of potassium infused was lowest in the group of patients receiving this rapid infusion, and there was no significant difference in the final plasma-potassium level between this $\mathrm{KCl}$ group and the two other KCl groups. Furthermore, no patients developed clinically significant hyperkalemia despite infusion of up to $366 \mathrm{mmol} \mathrm{KCl}$.

Antiarrhythmic drugs have previously been shown to increase the likelihood of conversion of ROAF to SR to approximately $70 \%$ within 12 hours ${ }^{6}$. Interestingly, the patients in our study receiving $\mathrm{KCl}$ infusion at a concentration of $15 \mathrm{mmol} / \mathrm{h}$ exhibited a similar conversion rate to SR (66.7\%) within a median infusion time of just 5.3 hours. Unfortunately, in $17 \%$ of the patients receiving $\mathrm{KCl}$ infusion at the highest infusion speed, the infusion had to be stopped due to pain at the infusion site. Of the remaining fast-infusion patients that completed the study protocol, the vast majority (82\%) converted to SR, and in the group of patients with an increase of plasma-potassium above median levels of increase, as many as $91.7 \%$ of these patients and all patients without a history of AF achieved conversion to SR. Furthermore, our results may suggest that $\mathrm{KCl}$ infusion is particularly effective in patients with the lowest 
1 plasma-potassium levels. Thus, in patients without potassium-induced infusion pain, rapidly 2 applied $\mathrm{KCl}$ may be as effective as other antiarrhythmic drugs and does not carry the risk or

3 burden of additional resources inherent with direct-current cardioversion therapy. We therefore suggest that patients with ROAF with plasma-potassium $\leq 4.0 \mathrm{mmol} / \mathrm{L}$ are candidates for receiving $\mathrm{KCl}$ infusion at $15 \mathrm{mmol} / \mathrm{h}$ for up to 12 hours. Indeed, of the patients in our study that experienced a rapid and substantial increase in circulating potassium, $75 \%$ converted to SR within this time. However, if a patient experience more than weak pain at the infusion site or if there is only a slight increase in plasma/serum-potassium, the $\mathrm{KCl}$ infusion should be terminated and another treatment strategy should be chosen.

None of our patients developed clinically significant hyperkalemia despite infusion of up to $366 \mathrm{mmol} \mathrm{KCl}$. This can be explained by the fact that the healthy kidney has a robust capacity to excrete potassium. Under normal conditions, most persons can ingest very large quantities of potassium (400 mmol per day or more) without clinically significant hyperkalemia. In the kidneys, potassium ingestion stimulates potassium secretion and inhibits potassium reabsorption ${ }^{20}$.

It is known that potassium chloride is vesicant and to avoid this, proper catheter position prior to and during infusion, in addition to a well-mixed infusion bag is important. Despite taking these steps, we were surprised that 12 of our patients reported pain at the infusion site, which was alleviated when the infusion was stopped. According to our protocol, staff were instructed to invert the infusion bag ten times after adding potassium chloride to the dextrose, to be sure that the solution was well-mixed. In addition, the protocol dictated that the peripheral vein catheter was to be placed in the antecubital vein and not at a more distal location. In some of the patients who experienced side-effects, the infusion bag may not have been well mixed, and in a few patients the peripheral vein catheter was placed on back of the hand, not following the study instructions. We suggest that in future investigations the solution should be pre-mixed by a pharmacist. 
1 It is uncertain whether magnesium infusion can promote conversion of AF to SR, as the

2 findings of two previous meta-analysis studies were contradictory ${ }^{21,22}$. Our patients in the

$3 \mathrm{KCl}$ group who exhibited serum magnesium levels $\leq 0.8 \mathrm{mmol} / \mathrm{L}$ also received magnesium

4 infusion since magnesium depletion reduces intracellular potassium concentration due to

5 renal potassium wasting ${ }^{23}$. However, few patients fit this criterion and received magnesium

6 infusion, and indeed, median serum magnesium level at the start of the protocol was $>0.80$

$7 \mathrm{mmol} / \mathrm{L}$ in all groups. In addition, magnesium infusion was not associated with altered conversion to SR, suggesting that inclusion of magnesium infusion in a subset of patients did not markedly affect the study results.

It could be argued that the placebo infusion with $50 \mathrm{mg} / \mathrm{mL}$ dextrose may decrease plasmapotassium and in this way augment the likelihood that the active arm of $\mathrm{KCl}$ infusion would work. However, the reduction of plasma-potassium in the placebo group was very small, with unchanged median values and only a small change in IQR from 3.6-4.0 mmol/L to 3.6-3.8 $\mathrm{mmol} / \mathrm{L}$. Thus, we believe this very small reduction in plasma-potassium had little effect on our results.

Seventy percent of the patients in the $\mathrm{KCl}$ group and $91 \%$ of the patients in the placebo group received heart rate-reducing medication after admission. Of these, most received metoprolol while only a few patients received verapamil or digoxin. Among the patients who converted to $\mathrm{SR}, 68 \%$ were treated with metoprolol in the $\mathrm{KCl}$ group vs. $82 \%$ in the placebo group, indicating that metoprolol did not contribute to the increased conversion rate in the $\mathrm{KCl}$ group.

At the post-discharge follow-up, we did not find any significant difference in the number of patients with AF between the KCL and placebo groups. However, this study had a low statistically power to detect such a difference. Moreover, since patients who did not convert to $\mathrm{SR}$ after $\mathrm{KCl}$ or placebo infusion were treated by direct-current cardioversion the day after the start of infusion, and since plasma- and serum-potassium levels at post-discharge follow- 
up were similar in the $\mathrm{KCl}$ and placebo groups, a significant difference in the frequency of $\mathrm{AF}$ is not to be expected. Whether potassium supplementation may have long-term effects in preventing AF in patients with circulating potassium in the lower normal range is not known and should be investigated in future studies.

The electrophysiological effects of potassium depend not only on its extracellular concentration but also on the direction (hypokalemia vs. hyperkalemia) and rate of change ${ }^{24}$. Gradually increasing plasma-potassium induces a biphasic electrical sequence, with initial augmentation and then depression of excitability, conduction, and automaticity ${ }^{25}$. Atrial muscle is more sensitive than ventricular muscle to elevated potassium levels, whereas specialized fibers such as in the sinoatrial node, internodal tracts, and the bundle of His are practically resistant ${ }^{26,27}$. The presently observed conversion of AF to SR upon increasing potassium levels could thus be due to a transient conduction acceleration which could terminate a re-entrant circuit in the atria ${ }^{14,28}$. Alternatively, return to SR may result from reduced triggered activity of atrial cardiomyocytes. Previous work in isolated ventricular myocytes has shown that hypokalemic conditions result in inhibition of the $\mathrm{Na}^{+}-\mathrm{K}^{+}$ATPase, and subsequent cellular calcium overload associated with generation of both early and delayed afterdepolarizations ${ }^{29,30}$. If similar pro-arrhythmic alterations occur in atrial myocytes, elevating blood potassium levels may favour a return to SR via a direct cellular action.

We measured plasma-potassium levels for the inclusion of patients, since the use of plasma is superior to serum for accurate determination of potassium ${ }^{31}$. While either plasma or serum can be used to assess potassium using an ion-selective electrode ${ }^{32}$, as presently employed, platelet release from platelets during the clotting process results in serum-potassium levels which are $0.36 \pm 0.18 \mathrm{mmol} / \mathrm{L}$ higher in serum than those measured in plasma ${ }^{31}$. This difference is in accordance with our findings of serum- and plasma-potassium levels measured at baseline (Table 4). 
2 Our study has several limitations. Firstly, due to slower than expected inclusion rate, five

3 fewer patients in the placebo group were recruited than planned based on the pre-study

\section{Limitations} power analysis. Secondly, since our results may indicate that $\mathrm{KCl}$ should be given at a speed of $15 \mathrm{mmol} / \mathrm{h}$, more significant results would have been expected if a greater number of patients had been assigned to this patient group. Thirdly, our beneficial findings are results of a post-hoc analysis and thus should be regarded as hypothesis-generating. Fourthly, although earlier studies have shown conflicting results regarding the ability of magnesium to convert ROAF to SR, we cannot rule out a confounding effect of magnesium in our study. However, few patients received magnesium infusion and the treatment was not associated with increased rate of conversion. Nevertheless, our study was not powered to address the effect of parallel infusion of magnesium and $\mathrm{KCl}$. Fifthly, due to our exclusion criteria it is possible that our results do not apply to the whole ROAF population.

\section{Conclusion}

Overall, our data do not demonstrate any increase in frequency or rate of conversion of ROAF to SR after increasing plasma-potassium levels in patients with levels in the lower normal range. However, in a post hoc analysis, we found that a rapid increase in circulating potassium may increase the likelihood of conversion of ROAF to SR. However, pain at the infusion site was relatively frequent and thus, not all patients with ROAF are able to complete such a treatment. Our findings are hypothesis driving and need to ultimately be reexamined in a larger study with higher statistical power. If verified, potassium's low-risk profile, electrophysiological effects and few contraindications could make it an adjunct or alternative to acute short-term conventional therapies used for conversion of ROAF.

\section{Sources of funding}

South-Eastern Norway Regional Health Authority. Grantnumber: 2013011

Declarations of interest: none. 


\section{References}

1. Kirchhof P, Benussi S, Kotecha D, Ahlsson A, Atar D, Casadei B, et al. 2016 ESC Guidelines for the management of atrial fibrillation developed in collaboration with EACTS. Eur Heart J 2016;37(38):2893-2962.

2. Hamilton A, Clark D, Gray A, Cragg A, Grubb N, Emergency Medicine Research Group E. The epidemiology and management of recent-onset atrial fibrillation and flutter presenting to the Emergency Department. Eur J Emerg Med 2015;22(3):155-61.

3. Vinson DR, Hoehn T, Graber DJ, Williams TM. Managing emergency department patients with recent-onset atrial fibrillation. J Emerg Med 2012;42(2):139-48.

4. Dell'Orfano JT, Patel H, Wolbrette DL, Luck JC, Naccarelli GV. Acute treatment of atrial fibrillation: spontaneous conversion rates and cost of care. Am J Cardiol 1999;83(5):788-90, A10.

5. Danias PG, Caulfield TA, Weigner MJ, Silverman DI, Manning WJ. Likelihood of spontaneous conversion of atrial fibrillation to sinus rhythm. J Am Coll Cardiol 1998;31(3):588-92.

6. Bonora A, Turcato G, Franchi E, Taioli G, Dilda A, Zerman G, et al. Efficacy and safety in pharmacological cardioversion of recent-onset atrial fibrillation: a propensity score matching to compare amiodarone vs class IC antiarrhythmic drugs. Intern Emerg Med 2017;12(6):853859.

7. Woods CE, Olgin J. Atrial fibrillation therapy now and in the future: drugs, biologicals, and ablation. Circ Res 2014;114(9):1532-46.

8. Nattel S, Opie LH. Controversies in atrial fibrillation. Lancet 2006;367(9506):262-72.

9. Krijthe BP, Heeringa J, Kors JA, Hofman A, Franco OH, Witteman JC, et al. Serum potassium levels and the risk of atrial fibrillation: the Rotterdam Study. Int J Cardiol 2013;168(6):5411-5.

10. Lu YY, Cheng CC, Chen YC, Lin YK, Chen SA, Chen YJ. Electrolyte disturbances differentially regulate sinoatrial node and pulmonary vein electrical activity: A contribution to hypokalemia- or hyponatremia-induced atrial fibrillation. Heart Rhythm 2016;13(3):781-8.

11. Weiss JN, Qu Z, Shivkumar K. Electrophysiology of Hypokalemia and Hyperkalemia. Circ Arrhythm Electrophysiol. 2018;10(3):e004667.

12. He FJ, MacGregor GA. Beneficial effects of potassium on human health. Physiol Plant 2008;133(4):725-35.

13. Manchester JH, Lamberti JJ, Jr. Reversion of atrial fibrillation following hyperkalemia. Chest 1970;58(4):399-402.

14. Mathew PK. Hyperkalemia-induced conversion of chronic atrial fibrillation to normal sinus rhythm; a case report. Angiology 1979;30(2):143-6.

15. Rothschild AH, Sridharan MR, Gondi B, Sohi GS, Flowers NC. Conversion of atrial fibrillation to normal sinus rhythm during hyperkalemia. J Ky Med Assoc 1985;83(6):295-8.

16. Gogas BD, Iliodromitis EK, Leftheriotis DI, Flevari PG, Rallidis LS, Kremastinos DT. Instantaneous electrocardiographic changes and transient sinus rhythm restoration in severe hyperkalaemia. Int J Cardiol 2011;148(2):e40-2.

17. Romeo E, D'Alto M, Santarpia G, Di Nardo G, Sarubbi B, Russo MG, et al. Hyperkalemiainduced conversion of permanent atrial fibrillation to normal sinus rhythm: a case report. J Cardiovasc Med (Hagerstown) 2011;12(9):678-80.

18. Santoro F, leva R, Spennati G, Ferraretti A, Franzese G, Tiscia G, et al. Tako-Tsubo cardiomyopathy and spontaneous cardioversion of permanent atrial fibrillation associated with acute hyperkalemia. Int J Cardiol 2013;167(3):e67-70.

19. Naccarelli GV, Dell'Orfano JT, Wolbrette DL, Patel HM, Luck JC. Cost-effective management of acute atrial fibrillation: role of rate control, spontaneous conversion, medical and direct current cardioversion, transesophageal echocardiography, and antiembolic therapy. Am J Cardiol 2000;85(10A):36D-45D. 
20. Gumz ML, Rabinowitz L, Wingo CS. An Integrated View of Potassium Homeostasis. N Engl J Med 2015;373(1):60-72.

21. Onalan O, Crystal E, Daoulah A, Lau C, Crystal A, Lashevsky I. Meta-analysis of magnesium therapy for the acute management of rapid atrial fibrillation. Am J Cardiol 2007;99(12):172632.

22. Ho KM, Sheridan DJ, Paterson T. Use of intravenous magnesium to treat acute onset atrial fibrillation: a meta-analysis. Heart (British Cardiac Society) 2007;93(11).

23. Gennari FJ. Hypokalemia. N Engl J Med 1998;339(7):451-8.

24. El-Sherif N, Turitto G. Electrolyte disorders and arrhythmogenesis. Cardiol J 2011;18(3):23345.

25. Fisch C, Knoebel SB, Feigenbaum H, Greenspan K. Potassium and the monophasic action potential, electrocardiogram, conduction and arrhythmias. Prog Cardiovasc Dis 1966;8(5):387-418.

26. de MW, Hoffman BF. Potassium ions and electrical activity of specialized cardiac fibers. Am J Physiol 1960;199:1125-30.

27. Vassalle M, Greenspan K, Jomain S, Hoffman BF. Effects of Potassium on Automaticity and Conduction of Canine Hearts. Am J Physiol 1964;207:334-40.

28. Ettinger PO, Regan TJ, Oldewurtel HA. Hyperkalemia, cardiac conduction, and the electrocardiogram: a review. Am Heart J 1974;88(3):360-71.

29. Aronsen JM, Skogestad J, Lewalle A, Louch WE, Hougen K, Stokke MK, et al. Hypokalaemia induces $\mathrm{Ca} 2+$ overload and $\mathrm{Ca} 2+$ waves in ventricular myocytes by reducing $\mathrm{Na}+, \mathrm{K}+-\mathrm{ATPase}$ $\alpha 2$ activity. J Physiol 2015;593(6):1509-1521.

30. Pezhouman A, Singh N, Song Z, Nivala M, Eskandari A, Cao H, et al. Molecular Basis of Hypokalemia-Induced Ventricular Fibrillation. Circulation 2015;132(16):1528-1537.

31. Nijsten MW, de Smet BJ, Dofferhoff AS. Pseudohyperkalemia and platelet counts. N Engl J Med 1991;325(15):1107.

32. Asirvatham JR, Moses V, Bjornson L. Errors in potassium measurement: a laboratory perspective for the clinician. N Am J Med Sci 2013;5(4):255-9. 